\title{
Expression of Egr3 in mouse gonads and its localization and function in oocytes
}

\author{
Hyejin Shin ${ }^{1, a}$, Dong-Won Seol ${ }^{2, a}$, Minyeong Nam', Haengseok Song ${ }^{2}$, \\ Dong Ryul Lee ${ }^{2, *}$, and Hyunjung Jade Lim ${ }^{1,3, *}$
}

\begin{abstract}
* Corresponding Authors: Dong Ryul Lee Tel: +82-31-881-7136, Fax: +82-31-881-7100, E-mail: drleedr@cha.ac.kr Hyunjung Jade Lim

Tel: +82-2-450-4087, Fax: +82-2-2030-7809,

E-mail: hlim@konkuk.ac.kr
\end{abstract}

'Department of Biomedical Science and Technology, Institute of Biomedical Science and Technology, Konkuk University, Seoul 05029, Korea

2 Department of Biomedical Science, CHA University, Seongnam 13884, Korea

${ }^{3}$ Department of Veterinary Medicine, Konkuk

University, Seoul 05029, Korea

a These authors contributed equally to this work. Submitted Oct 12, 2016; Revised Dec 2, 2016; Accepted Dec 7, 2016
Objective: The early growth response (Egr) family consists of four members (Egr1, Egr2, Egr3, and Egr4) that are zinc finger transcription factors. Among them, Egr3 is involved in transcriptional regulation of target genes during muscle spindle formation and neurite outgrowth. We previously showed that the immunoreactive Egr3 is localized on oocyte spindle and accumulate near the microtubule organizing center during meiosis I in mice. Egr3 was also shown to be localized on spermatocytes. We herein investigated if Egr3 is expressed in mouse gonads and if Egr3 blockade results in any defect in oocyte maturation.

Methods: Expression of Egr3 in mouse gonads was examined by reverse transcription-polymerase chain reaction. Full-length Egr3 and truncated Egr3 ( $\Delta$ Egr3) complementary RNAs (cRNAs) with Xpress tag at N-terminus and DsRed2 at C-terminus, and small interfering RNA (siRNA) targeting Egr3 were microinjected into mouse oocytes at germinal vesicle stage. Localization of microinjected Egr3 was examined by confocal live imaging and immunofluorescence staining.

Results: Egr3 mRNA was detected in mouse ovaries and testes from 1 to 4 week-old mice. An uncharacterized longer transcript containing 5 'untranslated region was also detected in 3 and 4 week-old gonads. Microinjected Xpress-Egr3-DsRed2 or Xpress- $\Delta$ Egr3-DsRed2 localized to nuclei and chromosomes during meiotic progression. Microinjection of these cRNAs or Egr3 siRNA in oocytes did not affect meiotic maturation. Immunofluorescence staining of Egr3 in Xpress- $\Delta$ Egr3-DsRed2-injected oocytes showed a positive signal only on meiotic spindle, suggesting that this antibody does not detect endogenous or exogenous Egr3 in mouse oocytes. Conclusion: The results show that Egr3 localizes to chromosomes during meiotic progression and that certain antibodies may not faithfully represent localization of target proteins in oocytes. Egr3 seems to be dispensable during oocyte maturation in mice.

Keywords: Mouse; Oocytes; Ovary; Testis; Egr3

\section{INTRODUCTION}

The early growth response (Egr) family of zinc finger transcription factors regulates critical genetic programs in cellular growth, differentiation, and function [1,2]. There are four Egr members, Egr1, Egr2, Egr3, and Egr4, and each member has specific and overlapping functions in a given physiological setting. Some Egr factors have shown to be involved in reproductive function. Egrl regulates expression of the luteinizing hormone beta subunit, and Egrl deficient mice suffer from female infertility resulting from hormone insufficiency [3]. Egr1 is induced by estrogen in the mouse uterus and expressed in stromal cells surrounding implanting blastocysts, suggesting a role for Egr1 in uterine biology [4]. Egr3 is implicated in neurodevelopment, learning and memory, immune response, and fibrogenic response [5-8], and this list shows functional diversity of this factor. As for Egr3 deficient mice, neurodevelopmental phenotypes are well described [5]. 
Whether Egr3 deficiency leads to other tissue-specific functions is just beginning to unravel with the availability of the Egr3 floxed mice [9]. In reproduction, expression of Egr3 in mouse oocytes and spermatocytes was reported by us [2]. In bovine granulosa cells, the administration of mycotoxin was shown to induce Egr3 expression [10]. Expression of Egr3 and Egr4 in the pig ovary is also recently reported [11]. In male mice, Egr4 exhibits a dynamic pattern of localization in germ cells and gonadal somatic cells depending on the stage of sexual maturity [12].

Previously, we showed that the immunoreactive Egr3 colocalizes with meiotic spindle and accumulates near cytosolic microtubule organizing centers (MTOCs) in oocytes during meiotic maturation. As a transcription factor, Egr3 was expected to localize to nucleus in cells and tissues, but localization of the immunoreactive Egr3 was noted in the cytoplasmic structures in mouse oocytes, early preimplantation embryos, and spermatocytes [2]. Several transcriptional regulators including Egr1 have been reported to exhibit similar localization on microtubuleassociated structures [13-15].

In this study, we elaborate further on the expression of Egr3 in gonads and its role in oocyte maturation. Whereas several pieces of information indicate involvement of Egr factors in female reproduction, it is not clear if Egr3 is required for oocyte maturation in mice. Thus, we herein investigated if functional blockade of Egr3 hinders meiotic maturation of oocytes by microinjecting dominant-negative Egr3 complementary RNA (cRNA) and small interfering RNA (siRNA) specific to Egr3. The results show that Egr3 is dispensable for meiotic maturation in mouse oocytes and that some of the commercially available anti-Egr3 antibodies may not faithfully represent endogenous and exogenous Egr3 proteins in oocytes.

\section{MATERIALS AND METHODS}

Mice

All mice were maintained in accordance with the policies of the Konkuk University Institutional Animal Care and Use Committee (IACUC). This study was approved by the Konkuk University IACUC (approval number KU15109). Mice were sacrificed after administration of avertin to minimize suffering. Four-week-old virgin ICR or BDF female mice were purchased from Orient-Bio (Seongnam, Korea).

\section{Collection and culture of oocytes}

Four-to-five-week-old virgin CrljOri:CD-1 (ICR) female mice were intraperitoneally injected with $7.5 \mathrm{IU}$ of pregnant mare's serum gonadotropin (Sigma-Aldrich, St. Louis, MO, USA) to induce folliculogenesis. Fully grown follicles were punctured $48 \mathrm{~h}$ later and cumulus-oocyte complexes were collected. The cumulus cells were then removed by using $300 \mu \mathrm{g} / \mathrm{mL}$ hyaluronidase (Sigma-Aldrich, USA) and germinal vesicle (GV) stage oocytes were retrieved in Quinn's Advantage Medium with HEPES (Sage BioPharma Inc., Bedminster, NJ, USA) containing 10\% substitute protein serum and $200 \mu \mathrm{M} 3$-isobutyl-1-methylxanthine (IBMX) (I5879; Sigma-Aldrich, USA). Cumulus cells were gently removed from the cumulus-oocyte complexes using a Pasteur glass pipette. The oocytes were incubated in microdrops of M16 medium (M7292; Sigma-Aldrich, USA) containing $200 \mu \mathrm{M}$ IBMX covered with mineral oil (Sage, USA) at $37^{\circ} \mathrm{C}$ under $5 \%$ $\mathrm{CO}_{2}$ in atmosphere until microinjection was conducted. After microinjection, these oocytes were placed in drops of M16 medium with $200 \mu \mathrm{M}$ IBMX for $8 \mathrm{~h}$. For oocyte maturation, these oocytes were placed in IBMX-free M16 medium for $20 \mathrm{~h}$. Rates of GV breakdown (GVBD) and polar body extrusion were monitored.

Reverse transcription-polymerase chain reaction

Reverse transcription-polymerase chain reaction (RT-PCR) was conducted as described previously [2]. Gonads were individually collected from 1, 2, 3, and 4 week-old female and male ICR mice. Each sample was subjected to RNA preparation by using TRI Reagent (Life Technologies, Invitrogen, Carlsbad, CA, USA) following the manufacturer's protocol. Isolated RNA $(2 \mu \mathrm{g})$ was subjected to reverse transcription (RT) using M-MLV reverse transcriptase (Beams Biotechnology, Seongnam, Korea). PCR was carried out using Prime Taq Premix (Genet Bio, Daejeon, Korea). Primer sequence are given in Table 1. PCR reaction was also performed for the mouse ribosomal protein L7 (Rpl7), a housekeeping gene. Annealing temperature is $59^{\circ} \mathrm{C}$ for all reactions. This experiments were repeated 6 times with independent samples.

\section{Plasmid constructs}

Full-length mouse Egr3 gene (NM_018781.3) was amplified by PCR from the mouse ovary cDNA sample [2]. To generate the dominant negative form of Egr3 [16], the truncated form of Egr3 (missing the first 249 amino acids, $\triangle$ Egr3) was generated by PCR from the full-length mouse Egr 3 cDNA. Full-length and truncated

Table 1. Primers used for reverse transcription-polymerase chain reaction

\begin{tabular}{llcl}
\hline Gene name & \multicolumn{1}{c}{ Sequence $\left.\mathbf{( 5}^{\prime}-\mathbf{3}^{\prime}\right)$} & Product size $(\mathbf{b p})$ & GenBank accession no. \\
\hline Egr3 & F: CAA TCT GTA CCC CGA GGA GAT & 204 & NM_018781.3 \\
Rpl7 & R: GCG AAC TTT CCC AAG TAG GTC & Variant: 257 & NM_001289925.1 \\
& F: TCA ATG GAG TAA GCC CAA AG & 246 & NM_011291.5 \\
\hline
\end{tabular}

$F$, forward; $R$, reverse. 
Egr3 DNAs were inserted into BglII and AgeI site of pDsRed2$\mathrm{N} 1$ vector (Clontech, Mountain View, CA, USA) and cloned into KpnI and XhoI site of pcDNA3.1/Myc-His vector (Invitrogen, USA). For in vitro transcription, full-length and truncated Egr3 construct containing Xpress tag at the N-terminus and DsRed2 at the C-terminus were amplified by PCR and cloned into BamHI and HindIII site of pRSET-A vector (Invitrogen, USA). The constructs are shown in Figure 1A, 1B.

\section{In vitro transcription and microinjection}

For live imaging of Egr3 expression in mouse oocytes, full-length and truncated Egr3 cRNAs were produced by in vitro transcription using mMessage mMachine $\mathrm{T} 7$ transcription kit (Ambion, Austin, TX, USA). To visualize microtubule in some experiments, $\beta$-tubulin-green fluorescent protein (GFP) cRNA was produced from pRN3- $\beta$-tubulin-GFP construct (a gift from Marie-Hélène Verlhac, CIRB, Collège de France, Paris, France) using T3 transcription kit. These RNAs were cleaned and concentrated by using RNeasy MinElute Clean up kit (Qiagen, Valencia, CA, USA).
These constructs were confirmed by in vitro translation by using TNT T7 Coupled Reticulocyte Lysate System kit (Promega, Madison, WI, USA). SMARTpool Egr3 siRNA (\#M-046392-010005) and non-targeting siRNA (\#D-001810-01-05) were purchased from Dharmacon (Lafayette, CO, USA). Knockdown capacity of this siRNA was first confirmed in 293T cells transfected with full-length Egr3 plasmid. Egr3 siRNA $(25 \mu \mathrm{M})$ was microinjected into GV oocytes and rates of GVBD and polar body extrusion were monitored.

To visualize Egr3 localization by live imaging, Egr3-DsRed2 or truncated Egr3-DsRed 2 cRNA $(0.0034 \mu \mathrm{M})$ was microinjected into GV oocytes. Injection volume was 5 to $10 \mathrm{pL}$, which is about $1 \%$ to $3 \%$ of total oocyte volume. Mouse oocytes were placed in drops of culture media containing $200 \mu \mathrm{M}$ IBMX for microinjection. Microinjection of cRNA or siRNA was performed using a Nikon (Nikon Corporation, Shinagawa-Ku, Tokyo, Japan) manipulator with picoinjector (Femtojet, Eppendorf, Hamburg, Germany) via pneumatic pressure.

A

The mouse Egr3 gene (NM_018781.3)
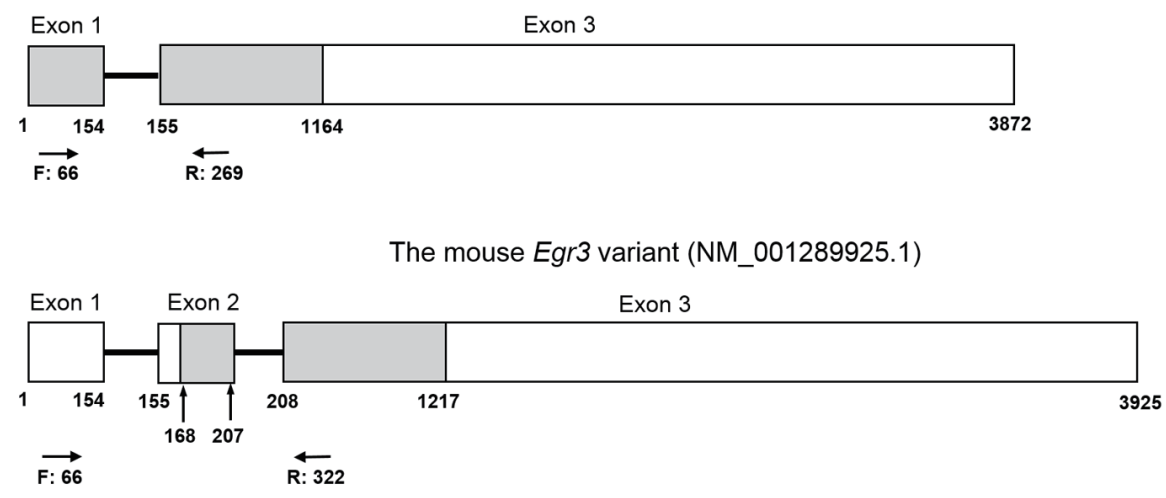

B
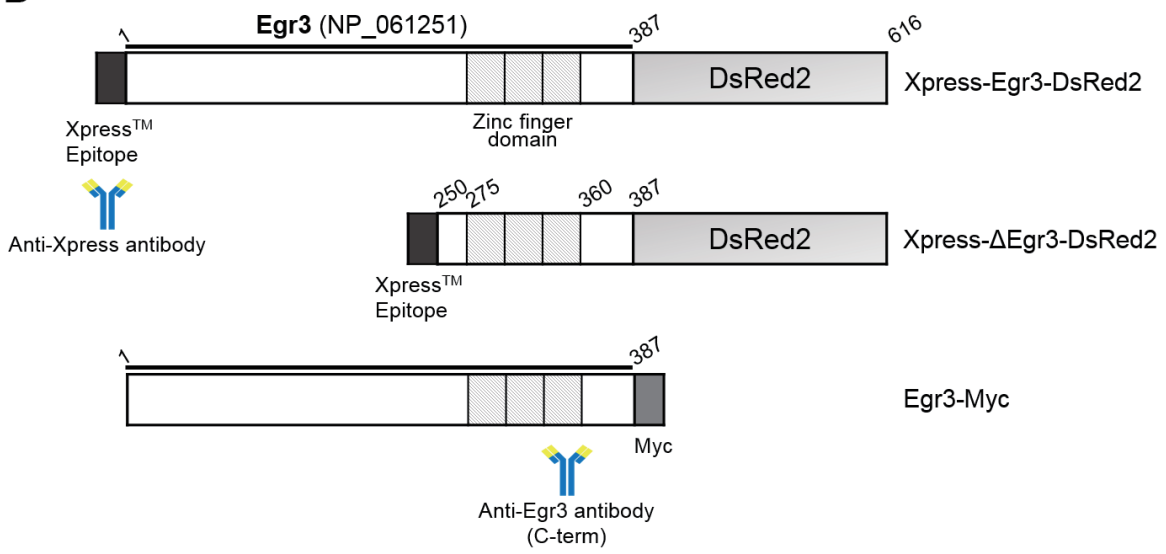

Figure 1. Diagrams depicting the structure of Egr3 gene and constructs used in this study. (A) The mouse Egr3 gene and the variant detected by RT-PCR. Coding regions are shown as gray shaded areas. Locations of forward (F) and reverse (R) primers are shown as arrows. The variant has another exon (exon 2) in the middle thus the PCR product is 53 bp longer. The predicted size of translated product is shorter because it uses a downstream start codon at 168. Gray box, translated region; while box, untranslated region; black line, intron. (B) Fusion constructs used in this study. Full-length and truncated ( $\Delta$ Egr3 without the first 249 amino acids) were cloned into pRSET vector containing Xpress epitope at the N-terminus. At the C-terminus, DsRed2 fluorescence tag was cloned in frame for live imaging. The locations of epitopes for the anti-Xpress and anti-Egr3 antibodies are shown. Egr3, early growth response protein 3; RT-PCR, reverse transcription-polymerase chain reaction. 


\section{Immunofluorescence staining}

The oocytes injected with Egr3 siRNA or Xpress- $\Delta$ Egr3-DsRed2 RNA were fixed in 3.7\% formaldehyde in phosphate-buffered saline (PBS) and permeabilized in $0.25 \%$ triton X-100 in PBS. They were blocked and incubated with $4 \mu \mathrm{g} / \mathrm{mL}$ antibody or 2 $\mu \mathrm{g} / \mathrm{mL}$ antibody of specific primary antibodies. Primary antibodies are the rabbit polyclonal anti-Egr3 antibody (sc-191; Santa Cruz Biotechnology, CA, USA) and the mouse anti-Xpress antibody (P/N 46-0528; Invitrogen, USA). Primary antibodies were probed with chick anti-rabbit IgG-Alexa Fluor 488 antibody and donkey anti-mouse IgG-Alexa Flour 488 antibody (1:250; Invitrogen, USA). DNA was counter-stained with TO-PRO-3-iodide (1:500; Invitrogen, USA). Rabbit IgG was used at the same concentration as a mock control in each experiment. Images were obtained using the Olympus Fluoview FV1000 spectral confocal microscope (Tokyo, Japan) and analyzed using the software Fluoview version 1.5, a platform associated with the confocal microscope.

\section{Protein extraction and western blotting}

293T cells were co-transfected with pcDNA3.1/Myc-Egr3 plasmid and Egr3 siRNA. Cells transfected with empty plasmid or nontargeting siRNA served as negative controls. Cell lysates were prepared in RIPA buffer (50 mM Tris [pH 7.5], $150 \mathrm{mM} \mathrm{NaCl}$, $1 \%$ Nonidet P-40, 0.5\% Na-deoxycholate, 0.1\% SDS, $1 \mathrm{mM}$ dithiothreitol (DTT), $1 \mathrm{mM}$ phenylmethylsulfonyl fluoride (PMSF), 1X Protease Inhibitor [Roche, Indianapolis, IN, USA]) and centrifuged at $13,000 \mathrm{rpm}$. The supernatant was subjected to bicinchoninic acid (BCA) assays (Thermo Scientific, Rockford, IL, USA) for quantitation. Fifty micrograms of protein were loaded per lane and separated in 10\% SDS-polyacrylamide gel electrophoresis (PAGE) gels. Western blotting was performed as previously described [2]. Anti- $\alpha$-tubulin (Sigma-Aldrich, USA) and anti-Egr3 (sc-191; Santa Cruz Biotechnology, USA) antibodies were used at 1:2,000 and 1:500, respectively. Anti-Myc antibody (\#631206; Clontech, USA) was used at 1:2,000. Horseradish peroxidase (HRP) -conjugated goat anti-rabbit and goat anti-mouse antibodies (GeneDEPOT, Barker, TX, USA) were used at 1:10,000. Super Signal West Femto ECL reagent (Thermo Scientific, USA) was used. Chemiluminescence signal was detected by LAS3000 (FUJIFILM, Tokyo, Japan).

\section{RESULTS}

Expression of Egr3 in ovaries and testes in mice

To examine if Egr3 is expressed in mouse gonads at different stages of sexual maturation, we collected ovaries and testes from $1,2,3$, and 4 week-old mice. The location of primers is depicted in Figure 1A. RT-PCR analysis showed that Egr3 mRNA is expressed in ovaries and testes in all groups. We also found that an uncharacterized longer transcript of Egr3 (NM_001289925.1) was present in 3 and 4-week-old gonads (Figure 2). Whether this form of Egr3 is functional or expressed in other tissues is not known.

\section{Microinjection of full-length and truncated Xpress-Egr3-}

DsRed2 does not hinder oocyte maturation

We previously showed that the immunoreactive Egr3 is specifically localized near MTOCs and on spindle during meiotic maturation of oocytes [2]. We examined the subcellular localization of Egr3 with live imaging after microinjecting full-length Egr3 cRNA. This construct has an Xpress tag at N-terminus and
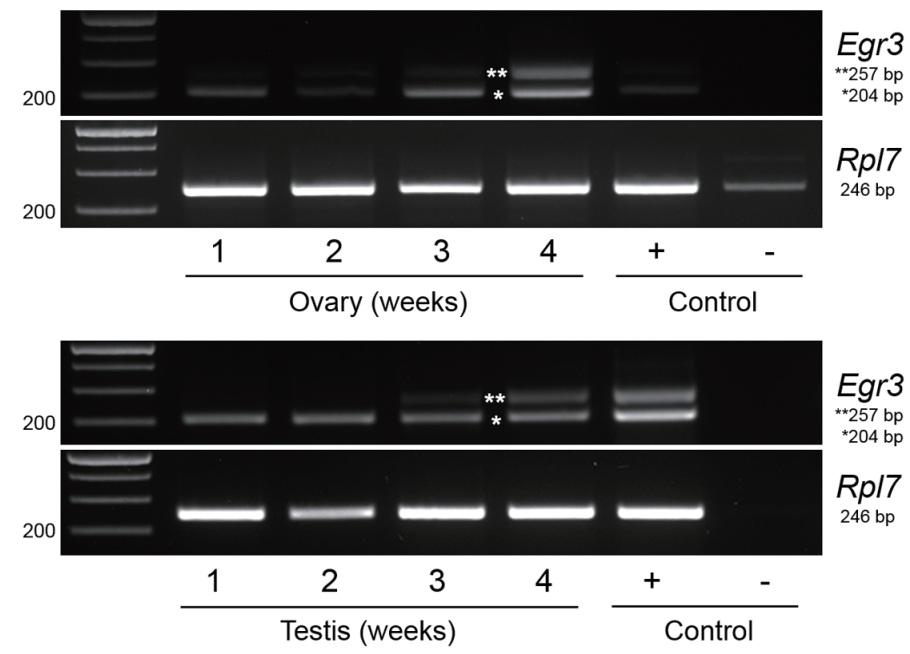

Figure 2. mRNA expression of the Egr3 gene in mouse ovaries and testes from 1 to 4 week-old mice. (A) Mouse ovaries from 1 to 4 week-old mice were subjected to RT-PCR. RNA from 4 week-old mouse testis was used as a positive control (+), and RNA from 4 week-old ovary without reverse transcription (RT) was used as a negative control (-). Expression of a housekeeping gene, Rpl7, was also examined. (B) Mouse testes from 1 to 4 week-old mice were subjected to RT-PCR. RNA from 4 week-old mouse ovary was used as a positive control (+), and RNA from 4 week-old testis without RT was used as a negative control (-). * ${ }^{*}$ Egr3; * * , a longer variant (NM_001289925.1). Egr3, early growth response protein 3; RT-PCR, reverse transcription-polymerase chain reaction; Rpl7, ribosomal protein L7. 
DsRed2 at C-terminus (Figure 1B). We also microinjected truncated Egr3 RNA (Xpress- $\Delta$ Egr3-DsRed2) lacking the first 249 amino acids, which is known as a dominant-negative form of Egr3 [16] (Figure 1B). As shown in Figure 3 by confocal live imaging, products of both Xpress-Egr3-DsRed2 and Xpress$\Delta$ Egr3-DsRed2 cRNAs localize to nucleus and chromosomes during meiotic maturation. We then monitored if microinjection of these Egr3 RNAs affect meiotic maturation rate in oocytes. Microinjection of either construct did not influence the rate of meiotic maturation (Table 2).

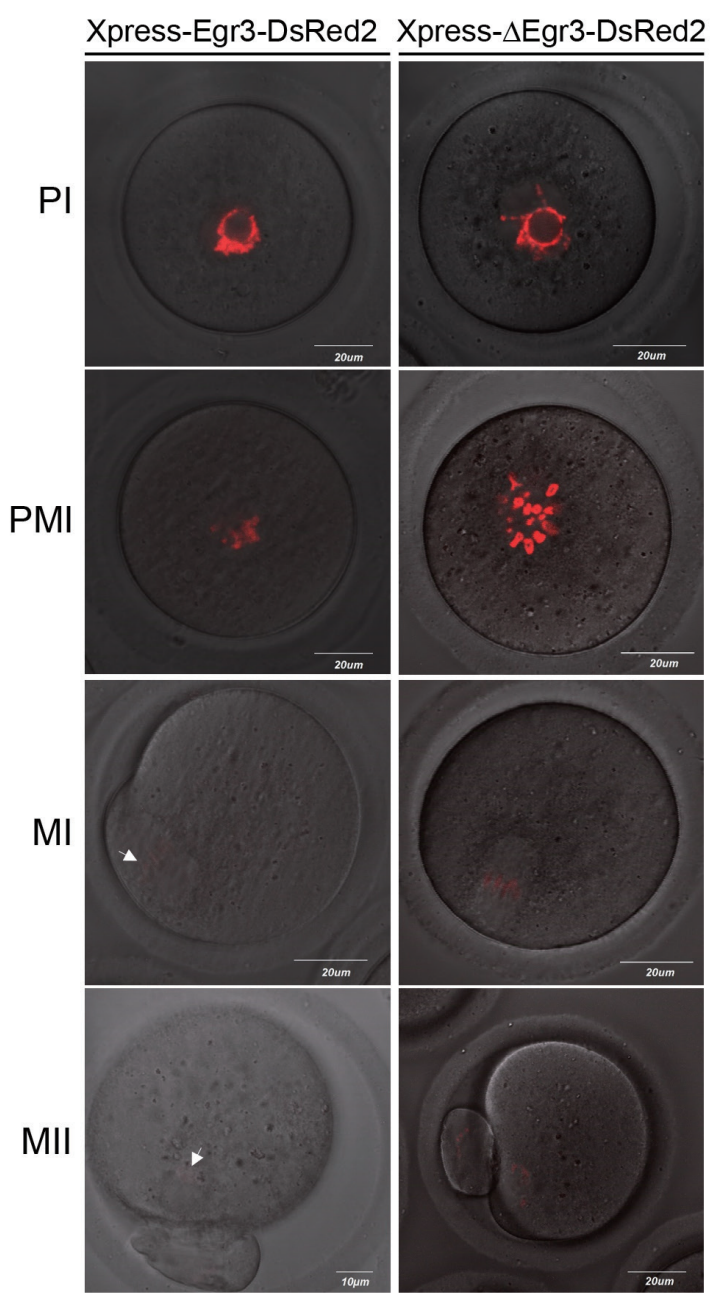

Figure 3. Microinjection of truncated Egr3 CRNA does not hinder oocyte maturation. Xpress-Egr3-DsRed2 or Xpress- $\triangle$ Egr3-DsRed2 cRNA $(0.0034 \mu \mathrm{M})$ was microinjected into GV oocytes in the presence of $200 \mu \mathrm{M}$ IBMX and oocytes were kept in M16 containing IBMX for $8 \mathrm{~h}$. They were then washed and cultured in IBMX-free M16 medium for $20 \mathrm{~h}$. Localization of microinjected constructs were monitored by confocal live imaging examining the expression of red fluorescent protein, DsRed2. During oocytes maturation, both Xpress-Egr3-DsRed2 or Xpress- $\triangle$ Egr3-DsRed2 proteins are localized in nuclei and chromosomes. Expression of Xpress-Egr3-DsRed2 protein is lower than that of Xpress- $\Delta$ Egr3-DsRed2, as translation efficiency of Xpress-Egr3DsRed2 is lower due to its length (about 750 bp longer than Xpress- $\triangle$ Egr3-DsRed2). White arrows indicate chromosomes. PI, prophase I; PMI, prometaphase I; MI, metaphase I; MII, metaphase II; Egr3, early growth response protein 3; GV, germinal vesicle; IBMX, 3-isobutyl-1-methylxanthine.
Table 2. Meiotic maturation rate of oocytes injected with Xpress-Egr3-DsRed2 or Xpress- $\triangle$ Egr3-DsRed2 (RNA ${ }^{11}$

\begin{tabular}{lccc}
\hline \multirow{2}{*}{ Group } & \multirow{2}{*}{$\begin{array}{c}\text { Total } \\
\text { oocytes }\end{array}$} & \multicolumn{2}{c}{ Oocytes maturation rate (\%) } \\
\cline { 3 - 4 } & & GVBD oocytes (\%) & MII oocytes (\%) \\
\hline Xpress-Egr3-DsRed2 & 85 & $84(98.82)$ & $51(60.00)$ \\
Xpress- $\Delta$ Egr3-DsRed2 & 78 & $74(94.87)$ & $52(66.66)$ \\
Water injection & 40 & $40(100)$ & $29(72.50)$ \\
No injection & 68 & $65(95.59)$ & $54(79.41)$ \\
\hline
\end{tabular}

GVBD, germinal vesicle breakdown; MII, metaphase II; IBMX, 3-isobutyl-1-methylxanthine.

1) Microinjection was performed in the presence of IBMX and oocytes were incubated in M16 medium containing $200 \mu \mathrm{M}$ IBMX for $8 \mathrm{~h}$. They were then cultured in IBMX-free M16 medium for $20 \mathrm{~h}$. GVBD was monitored after 3 to $5 \mathrm{~h}$ of culture and polar body extrusion (MII) after $20 \mathrm{~h}$ from the beginning of IBMX-free culture.

Meiotic maturation is not hindered by Egr3 siRNA in oocytes We next tested if microinjection of Egr3 siRNA has any influence on meiotic maturation. The specificity of Egr 3 siRNA was first confirmed by western blotting in $293 \mathrm{~T}$ cells transfected with Egr3-Myc plasmid (Figure 4A). However, microinjection of Egr3 siRNA to GV oocytes did not affect GVBD or polar body formation (Table 3). Spindle forms normally in metaphase I (MI) oocytes injected with Egr3 siRNA, as evidenced by normal distribution of tubulin-GFP (Figure 4B).

Egr3 localization: potential artefact in immunolocalization The observation that the microinjected Xpress- $\Delta$ Egr3-DsRed2 constructs are only confined to nuclei and chromosomes contradicts our previous observation that immunoreactive Egr3 localized to MTOCs and microtubules [2]. Thus, we side-by-side compared localization of Egr3 in microinjected oocytes by confocal live imaging, and anti-Xpress and anti-Egr3 immunofluorescence staining in fixed oocytes. As shown in Figure 5, DsRed2 signal is clearly confined to chromosomes. Anti-Xpress antibody detected a diffuse pattern of signal on or near metaphase chromosomes. This pattern is less clear than DsRed2 live imaging, but some signal overlaps with DNA. In contrast, anti-Egr3 antibody shows positive signal only on meiotic spindle (Figure 5). This anti-Egr3 antibody does not detect exogenous Xpress- $\Delta$ Egr3DsRed2, because the signal is negative on chromosomes. This

Table 3. Meiotic maturation rate of oocytes injected with Egr3 siRNA or nontargeting siRNA ${ }^{1)}$

\begin{tabular}{lccc}
\hline \multirow{2}{*}{ Group } & \multirow{2}{*}{$\begin{array}{c}\text { Total } \\
\text { oocytes }\end{array}$} & \multicolumn{2}{c}{ Oocytes maturation rate (\%) } \\
\cline { 3 - 4 } & & GVBD oocytes (\%) & MII oocytes (\%) \\
\hline Egr3 siRNA & 167 & $164(98.20)$ & $119(71.26)$ \\
Non-targeting siRNA & 155 & $146(94.19)$ & $116(74.84)$ \\
No injection & 63 & $62(98.41)$ & $45(71.42)$ \\
\hline
\end{tabular}

GVBD, germinal vesicle breakdown; MII, metaphase II; IBMX, 3-isobutyl-1-methylxanthine.

${ }^{1)}$ Microinjection was performed in the presence of IBMX and oocytes were incubated in M16 medium containing $200 \mu \mathrm{M}$ IBMX for $8 \mathrm{~h}$. They were then cultured in IBMX-free M16 medium for $20 \mathrm{~h}$. GVBD was monitored after 3 to $5 \mathrm{~h}$ of culture and polar body extrusion (MII) after $20 \mathrm{~h}$ from the beginning of IBMX-free culture. 
A

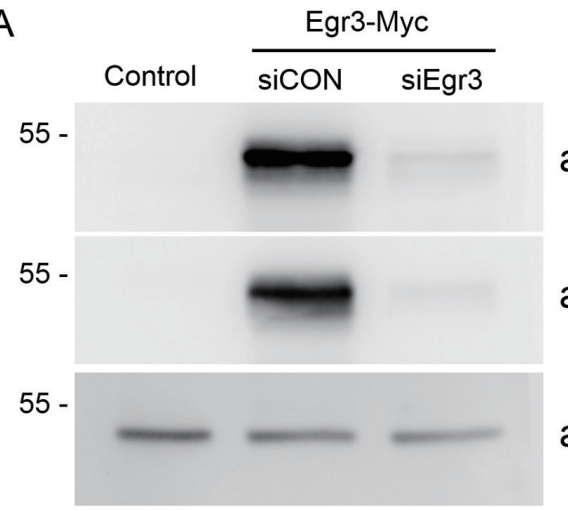

\section{anti-Egr3}

anti-Myc

anti-a-tubulin

B

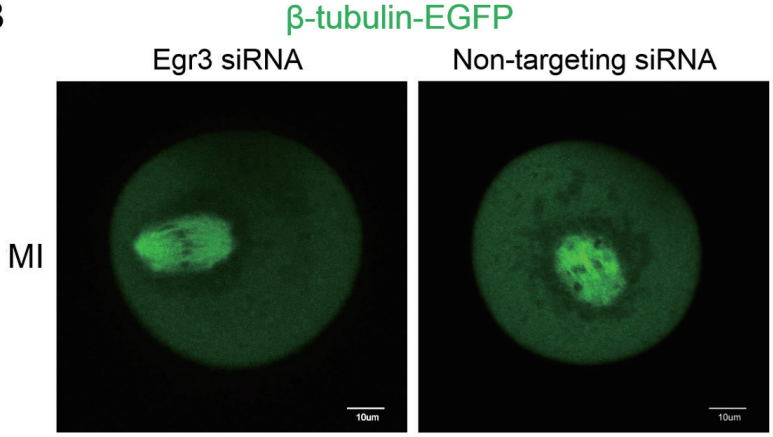

Figure 4. Microinjection of Egr3 siRNA does not hinder oocyte maturation. (A) Western blot analysis of Egr3 in 293T cell co-transfected with pcDNA3.1/myc-hisEgr3 (Egr3-Myc) and Egr3 siRNA (siEgr3) or non-targeting siRNA (siCON). Empty plasmid served as a negative control (Control). These cells were subjected to western blot analysis with anti-Egr3 antibody. Fifty micrograms of cell lysates were run on $10 \%$ SDS-PAGE gels. (B) Microinjection of Egr3 siRNA in GV oocytes does not interfere with meiotic maturation. Confocal live image of meiotic spindle after coinjection of $0.5 \mu \mathrm{g} / \mu \mathrm{L} \beta$-tubulin-EGFP cRNA and $25 \mu \mathrm{M}$ Egr3 siRNA or non-targeting siRNA. After microinjection, the oocytes were incubated in M16 medium supplemented with $200 \mu \mathrm{M}$ IBMX for $8 \mathrm{~h}$, then they were washed and cultured in IBMX-free M16 medium for $6 \mathrm{~h}$. Spindle forms normally in both groups. Ml, metaphase I; Egr3, early growth response protein 3; SDS-PAGE, SDS-polyacrylamide gel electrophoresis; GV, germinal vesicle; EGFP, enhanced green fluorescent protein; IBMX, 3-isobutyl-1-methylxanthine. result suggests that spindle-positive signal detected by anti-Egr3 antibody seems to be nonspecific.

\section{DISCUSSION}

In this study, we show that mouse gonads express Egr3 mRNA, both at immature and sexually maturing stages of age. Herein we present experiments to test functionality of Egr3 in mouse oocytes, providing evidence that Egr3 is dispensable for meiotic maturation in mouse oocytes.

In RT-PCR analysis, a bigger, uncharacterized Egr3 isoform was noted in 3 and 4-week ovaries. This variant, NM_001289925.1, has another exon inserted between two original exons of the mouse Egr3 gene (Figure 1A). This form using a downstream start codon is expected to produce a shorter product with distinct N-terminus. Several isoforms of Egr3 have been reported in brain and they seem to have different transcriptional activity $[17,18]$. Interestingly, the appearance of this variant is associated with sexual maturation of the ovary. Whether this particular variant in mouse gonads is expressed in brain or other tissues and functionally active is currently unknown.

Egr3 without the $\mathrm{N}$-terminal half of the protein is known as a dominant-negative form which blocks activity of Egr factors [16]. Commercially available siRNA specific to Egr3 efficiently downregulates translation of Egr3 (Figure 4A). Microinjection of either dominant-negative Egr3 or Egr3 siRNA did not influence the rates of GVBD and $\mathrm{PB}$ extrusion, suggesting that Egr3 is functionally dispensable during oocyte maturation (Tables 2, 3). During the course of this study, we noticed that Xpress-Egr3DsRed2 and Xpress- $\Delta$ Egr3-DsRed2 proteins are confined only on nuclei and chromosomes after microinjection when observed by live imaging (Figure 3). This is in discrepancy with our previous observation that anti-Egr3 antibody produced signal near MTOC and on spindle of maturing oocytes. In our previous

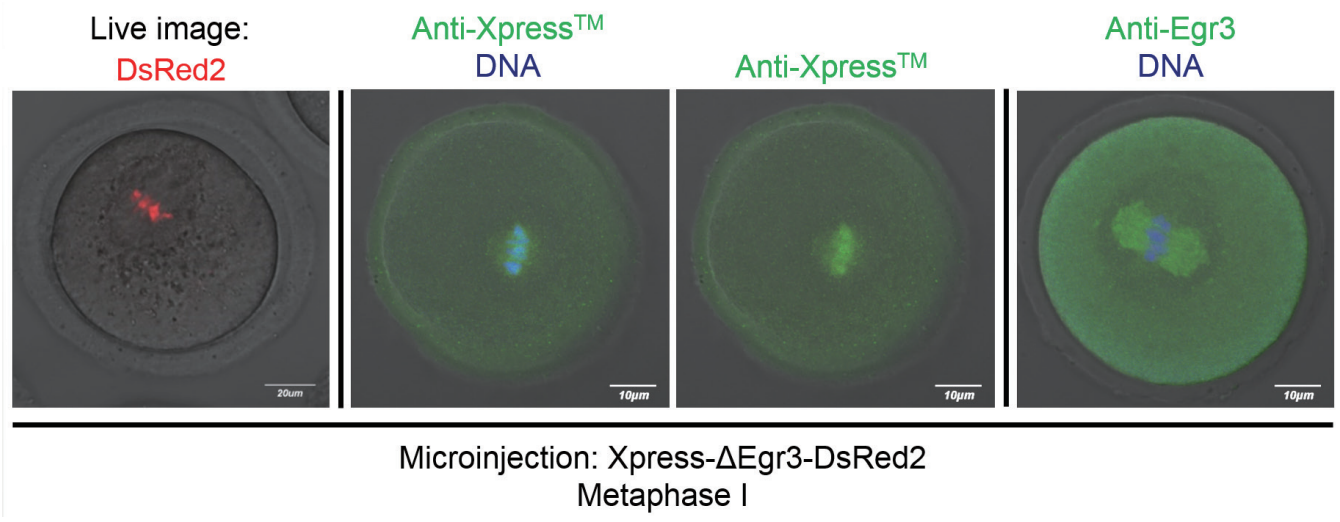

Figure 5. Various methods of validating Egr3 localization in mouse oocytes and potential artefacts. Confocal live imaging and immunofluorescence staining with anti-Xpress and anti-Egr3 antibodies were compared in MI oocytes injected with Xpress- $\Delta$ Egr3-DsRed2 CRNA. GV oocytes were microinjected with Xpress- $\Delta$ Egr3-DsRed2 cRNA under IBMX and incubated for $8 \mathrm{~h}$ in M16 medium supplemented with IBMX. For oocyte maturation, these oocytes were placed in IBMX-free M16 medium for $8 \mathrm{~h}$. At Ml stage, o0cytes were either subjected to confocal live imaging or immunofluorescence staining with indicated antibodies. Egr3, early growth response protein 3; Ml, metaphase I; GV, germinal vesicle; IBMX, 3-isobutyl-1-methylxanthine. 
study, we had tested five commercially available anti-Egr3 antibodies and two of them had produced the observed pattern of localization in oocytes [2] (Figure 5). Both antibodies, detecting C-terminal region of Egr3 protein, detect Egr3 in western blotting and recognize exogenous Egr3 in nuclei of PC12 or MCF7 cells. Interestingly, one of these antibodies also detect immunoreactive Egr3 in growing neurites of PC12 cells (data not shown). Thus, certain anti-Egr3 antibody recognizes microtubule-based subcellular structures both in PC12 and mouse oocytes; neurite and meiotic spindle. The similarity between these structure is evident, i.e., they both utilize microtubule. However, our side-by-side comparison in Figure 5 shows that anti-Egr3 antibody produces a distinct pattern of subcellular localization compared to live imaging and anti-Xpress immunofluorescence staining. AntiXpress antibody detecting Xpress tag on the fusion construct (Figure 1B) produced a signal much closer to Egr3-DsRed2 live imaging (Figure 5), although the pattern was more diffused than that of DsRed2 live imaging. While we vigorously validated the specificity of antibodies in our previous investigation [2], it is possible that this anti-Egr3 antibody might produce artefactual pattern of localization in oocytes. Antibodies are often the most common culprits of problems in biological experiments due to non-specific binding to other proteins [19]. Whether this particular anti-Egr3 antibody detects other target(s) or processed form of Egr3 protein is not clear and it requires further validation.

\section{CONFLICT OF INTEREST}

We certify that there is no conflict of interest with any financial organization regarding the material discussed in the manuscript.

\section{ACKNOWLEDGMENTS}

This study was supported by Basic Science Research Program through the National Research Foundation of Korea (NRF) funded by the Ministry of Education, Science and Technology (NRF-2013 R1A1A2059221). This paper was also written as part of Konkuk University's research support program for its faculty on sabbatical leave in 2016.

\section{REFERENCES}

1.O'Donovan KJ, Tourtellotte WG, Millbrandt J, Baraban JM. The EGR family of transcription-regulatory factors: progress at the interface of molecular and systems neuroscience. Trends Neurosci 1999;22: 167-73.

2.Shin H, Kwon S, Song H, Lim HJ. The transcription factor Egr3 is a putative component of the microtubule organizing center in mouse oocytes. PLoS One. 2014;9:e94708.

3.Lee SL, Sadovsky Y, Swirnoff AH, et al. Luteinizing hormone deficiency and female infertility in mice lacking the transcription factor NGFI-A (Egr-1). Science 1996;273:1219-21.
4.Kim HR, Kim YS, Yoon JA, et al. Egr1 is rapidly and transiently induced by estrogen and bisphenol A via activation of nuclear estrogen receptor-dependent ERK1/2 pathway in the uterus. Reprod Toxicol 2014;50:60-7.

5.Tourtellotte WG, Milbrandt J. Sensory ataxia and muscle spindle agenesis in mice lacking the transcription factor Egr3. Nat Genet 1998;20:87-91.

6.Li L, Yun SH, Keblesh J, et al. Egr3, a synaptic activity regulated transcription factor that is essential for learning and memory. Mol Cell Neurosci 2007;35:76-88.

7.Li S, Miao T, Sebastian M, et al. The transcription factors Egr2 and Egr3 are essential for the control of inflammation and antigen-induced proliferation of B and T cells. Immunity 2012;37:685-96.

8.Fang F, Shangguan AJ, Kelly K, et al. Early growth response 3 (Egr-3) is induced by transforming growth factor- $\beta$ and regulates fibrogenic responses. Am J Pathol. 2013;183:1197-208.

9.Quach DH, Oliveira-Fernandes M, Gruner KA, Tourtellotte WG. A sympathetic neuron autonomous role for Egr3-mediated gene regulation in dendrite morphogenesis and target tissue innervation. J Neurosci 2013;33:4570-83.

10. Guerrero-Netro HM, Chorfi Y, Price CA. Effects of the mycotoxin deoxynivalenol on steroidogenesis and apoptosis in granulosa cells. Reproduction 2015;149:555-61.

11. Wang J, Zhao Y, Gu K, et al. The novel porcine gene early growth response 4 (Egr4) is differentially expressed in the ovaries of Erhualian and Pietrain pigs. Reprod Fertil Dev 2014;26:587-98.

12. Hogarth CA, Mitchell D, Small C, Griswold M. EGR4 displays both a cell- and intracellular-specific localization pattern in the developing murine testis. Dev Dyn 2010;239:3106-14.

13. Mora GR, Olivier KR, Cheville JC, et al. The cytoskeleton differentially localizes the early growth response gene-1 protein in cancer and benign cells of the prostate. Mol Cancer Res 2004;2:115-28.

14. Soubry A, Staes K, Parthoens E, et al. The transcriptional repressor Kaiso localizes at the mitotic spindle and is a constituent of the pericentriolar material. PLoS One 2010;5:e9203.

15. Wei H, Wang Q, Du J, et al. Unique subcellular distribution of RPB1 with a phosphorylated C-terminal domain (CTD) in mouse oocytes during meiotic division and its relationship with chromosome separation. J Reprod Dev 2015;61:541-8.

16. Levkovitz Y, O'Donovan KJ, Baraban JM. Blockade of NGF-induced neurite outgrowth by a dominant-negative inhibitor of the egr family of transcription regulatory factors. J Neurosci 2001;21:45-52.

17. O'Donovan KJ, Baraban JM. Major Egr3 isoforms are generated via alternate translation start sites and differ in their abilities to activate transcription. Mol Cell Biol 1999;19:4711-8.

18. O'Donovan KJ, Levkovitz Y, Ahn D, Baraban JM. Functional comparison of Egr3 transcription factor isoforms: identification of an activation domain in the $\mathrm{N}$-terminal segment absent from Egr3beta, a major isoform expressed in brain. J Neurochem 2000;75:1352-7.

19. Baker M. Reproducibility crisis: Blame it on the antibodies. Nature 2015;521:274-6. 